\section{Einsparpotential von 40 Prozent}

\section{Von den jährlich in Deutschland anfallenden 1,5 Millionen Tonnen Elektronik- schrott geht ein nicht unerheblicher Teil auf alte Fernseher zurück. In einem Verbundprojekt von TV-Geräteherstellern und dem Öko-Institut wird vor die- sem Hinfergrund die Entwicklung eines "Grünen Fernsehers" vorangetrieben, der sowohl zu weniger Schrott und Sonderabfällen führt als auch eine opti- mierte Produkłökobilanz vorweisen kann.}

$\mathrm{D}$

Von Volker Strubel gen aus gebrauchten Elektro- und Elektronikgeräten sowie der Entwurf der sogenannten Elektronikschrottverordnung machen die Suche nach neuen Lösungswegen dringend notwendig. Dies gilt speziell für TV-Gerätehersteller. Jährlich wandern in der Bundesrepublik rund 5,7 Millionen Fernseher über den Ladentisch. 1994 gab es ein Elektronikschrottaufkommen von rund 1,5 Millionen Tonnen, darunter etwa drei Millionen Fernsehgeräte.

Spätestens an dieser Stelle beginnt das Problem. Jedes Gerät besteht aus mehr als 2000 Bauteilen. In diese Bauteile gehen wiederum schätzungsweise mehrere tausend Einzelstoffe ein. Der Materialmix mit zahlreichen giftigen Stoffen macht die Entsorgung schwierig. Hier setzt das Projekt „Entwicklung eines Grünen Fernsehers" an, das ein Verbundprojekt der TVGerätehersteller Grundig, Loewe Opta, Philips, Nokia sowie Deutsche Thomson-Brandt und dem Öko-Institut e.V. ist und das vom Deutschen Bundesministerium für Bildung, Wissenschaft, Forschung und Technologie (BMBF) gefördert wird. Dabei soll sich das Forschungsprojekt nicht auf Fernseher beschränken. Mittelfristiges Ziel ist es, die Erkenntnisse auf andere komplexe Elektronik-Produkte zu übertragen. TV-Geräte bieten sich als Grundlage besonders an, weil hier praktisch alle in der Elektronik vorkommenden Aufgabenstellungen vereint sind. Dem ,grünen Fernseher“ könnte z.B. ein "grüner" Computer oder Fotokopierer folgen. Dies wird auch verstärkt dadurch erreicht, daß es sich bei diesem Projekt um ein Verbundvorhaben von verschiedenen Herstellern und einem Forschungsinstitut handelt. Somit können spezifisches Know-How ausgetauscht sowie Synergieeffekte genutzt werden und die Bündelung von Nachfragekapazitäten fördert Kooperationen der Akteure mit Zulieferern und Entsorgern.

\section{Ergebnisse der Vorphase}

Zur Ermittlung dieser Ergebnisse wurde ein virtuelles Referenzgerät definiert, das den derzeitigen Stand der Technik - also das durchschnittliche TV-Gerät, dessen Bauteile und Einzelbestandteile, Lebensdauer etc. repräsen-
Quelle: Ōko-lnstitut: Green IV. Enwicklung eines grünen Fernsehers. 1996. tiert. Ebenso fanden die dazugehörigen Referenzrahmenbedingungen wie Nutzungsdauer, Einschaltgewohnheiten und der gängigen abfallwirtschaftlichen Praxis Eingang in die Definition des virtuellen Referenzgerätes. Um potentielle ökologische Optimierungspotentiale zu ermitteln, wurde zudem ein virtueller Referenzprototyp definiert und versucht, von beiden Typen Ökobilanzen der Einzelteile zu erstellen. Ausgehend von Datenrecherchen bei den beteiligten Firmen und Zulieferern wurden eine Reihe von orientierenden Ökobilanzen für Einzelmaterialien und Bauteile erstellt. Dabei zeigte sich, daß noch erhebliche Datenlücken bei bestimmten Produktionsprozessen und bei den geplanten Prozeßinnovationen bestehen.

Weitere zentrale Ergebnisse der jetzt abgeschlossenen einjährigen Vorphase sind (siehe auch Grafik):

- Gerade für den Energieverbrauch erwies es sich bei Fernsehern als entscheidend, wieviel Stunden er täglich läuft. Bei drei Stunden täg-

Ressourcenverbrauch
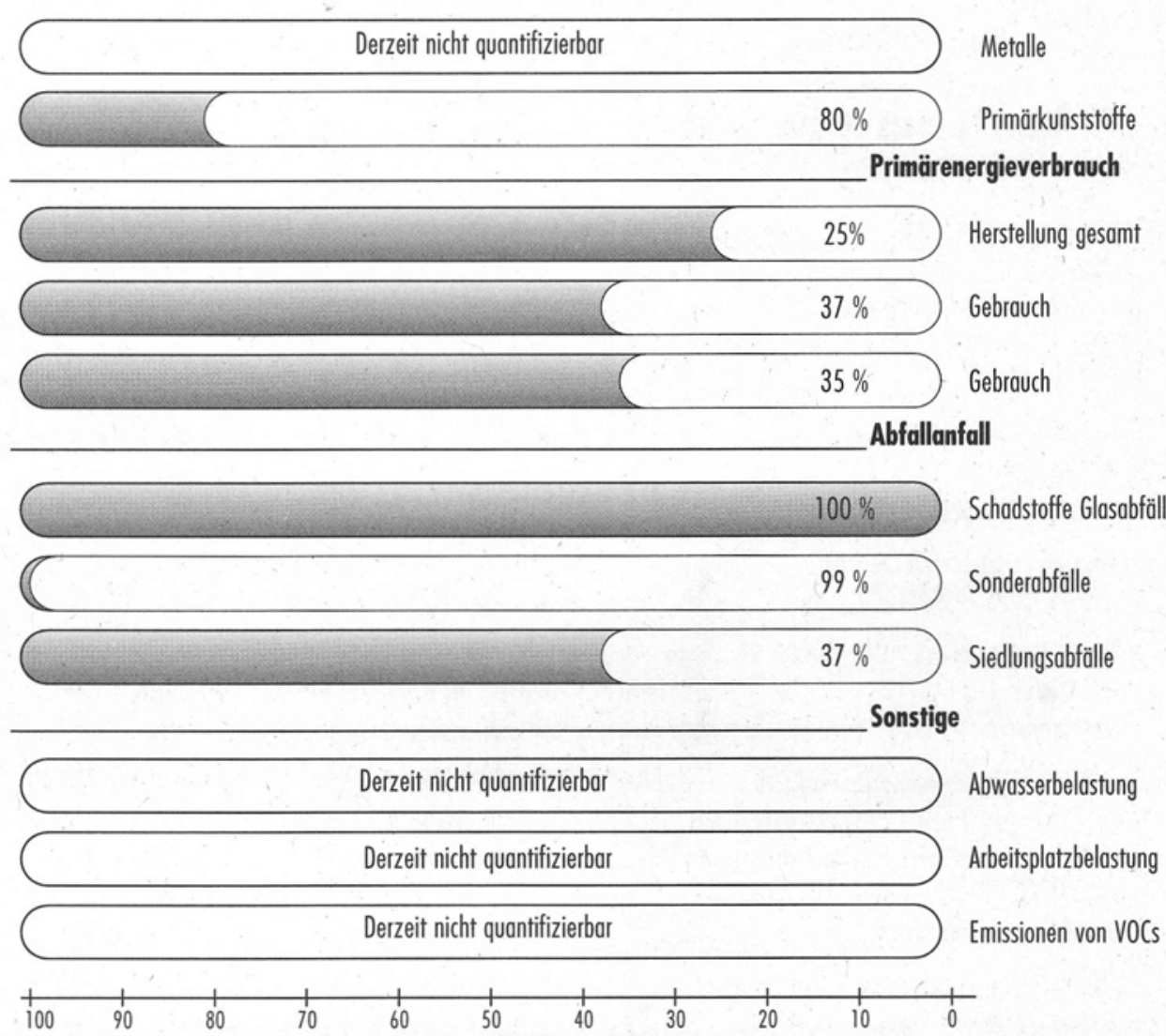

Zusommenfossende Darstellung der Umweltentlostungspotentiole der verfolgten Technologiekonzepte in der Vorphose 


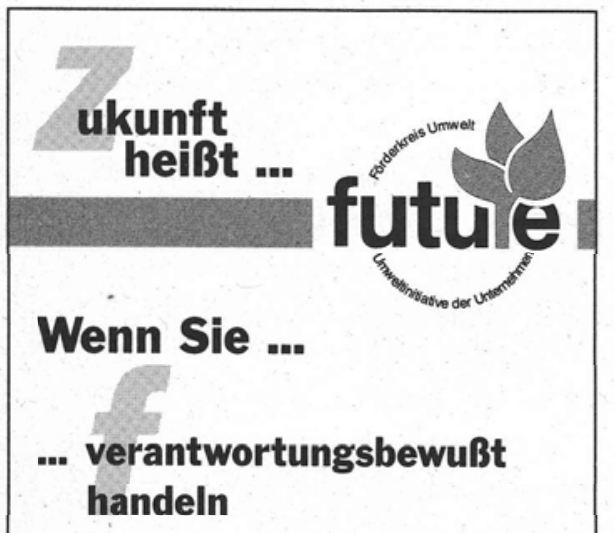

\section{... ökologisch sinnvoll wirtschaften}

\section{... kreative Konzepte entwickeln}

\section{... eine umweltorientierte und sozial verpflichtete Marktwirtschaft gestalten \\ wollen, dann machen}

\section{Sie mit uns}

\section{Zukunft zur Chance!}

Denn der Förderkreis Umwelt future e.V. - eine Umweltinitiave engagierter Unternehmer steht für einen effektiven betrieblichen Umweltschutz und entwickelt die dazu notwendigen Instrumente.

\section{Ja, senden Sie uns}

○ ausführliche Informationen

0 ein Probeexemplar der futureZeitschrift "Unternehmen und Umwelt"

\section{Firma}

\section{PLZ/Ort}

Straße/Nr.

Tel./Ansprechpartner

Förderkreis Umwelt future e.V.

Kollegienwall 22 a

49074 Osnabrück

Tel.: 0541/2 8784

Fax:: 0541/2 8304 lich überwiegt beispielsweise der Energieverbrauch der Gebrauchsphase mit fast 90 Prozent. Bei niedrigen Beitriebszeiten wird die Bedeutung der Herstellung größer.

- Durch technologische Neuerungen sowohl bei der Geräteherstellung als auch in der Gebrauchsphase lassen sich beachtliche Umweltentlastungen erwarten.

- Selbst bei sehr günstig angenommenen Rahmenbedingungen ist das maximal erreichbare Entlastungspotential durch aufbereitete Sekundärmaterialien in der Neugeräteherstellung mit insgesamt rund 25 Prozent eher gering.

- Sonderabfälle und schadstoffhaltiger Müll lassen sich durch eine gezielte Erfassung von Altgeräten und entsprechender Verwertung von Gerätekomponenten fast völlig vermeiden.

- Siedlungsmüll, der im Rahmen der Produktion anfällt, kann um mehr als 35 Prozent verringert werden.

- Für den gesamten Lebensweg eines TV-Gerätes von der Herstellung, Vertrieb, Bebrauch bis zur Entsorgung ermittelte das Öko-Insitut ein Einsparpotential von rund 40 Prozent.

\section{Zeitplanung}

In der mittlerweile angelaufenen Hauptphase des Projekts geht es darum, die in der Vorphase ermittelten Einsparpotentiale systematisch zu erschließen und anhand von Musterbeispielen erstmals zu realisieren. Zu Ende der Hauptphase im September 1998 soll deshalb auf Basis der wissenschaftlichen und technischen Erkenntnisse ein funktionsfähiger Prototyp vorgestellt werden, der neben den Kriterien Funktion, Sicherheit, Design und Wirtschaftlichkeit auch die Umweltverträglichkeit hinsichtlich des gesamten Produktlebensweges optimal erfüllt. Um dies zu ermöglichen, werden derzeit in folgenden Bereichen neue Technlogien entwickelt:

- Neue Technolgien für Schaltungsträger als Ersatz der Leiterplatte,

- Optimierung von elektrischen Bauelementen,

- Verbesserung von Lautsprechern,

- Schaltungstechnik zu Verkleinerung elektrischer Bauelemente und

- Optimierung des Bildröhrenrecyclings.

Neben den technologischen Entwicklungen werden Konzepte zur Verlängerung der Lebensdauer und nachträglichen Modernisierungen der Geräte sowie für eine Reduktion des Energieverbrauchs in der Gebrauchsphase erarbeitet.
Grundsätzlich werden zwei unterschiedliche Modellvarianten verfolgt. Das Metallmodell zielt auf den größtmöglichen Einsatz von Metallen und somit auf Ressourcenschonung durch einfache Demontage und rohstoffliche Verwertung. Das Kunststoffmodell stützt sich aufgrund deren guter werkstofflicher Verwertbarkeit auf thermoplastische Kunststoffe.

\section{Aufgabenverteilung}

Das Vollkunststoffkonzept wird gemeinsam von Grundig und Thomson bearbeitet. Zur Verbesserung der Kreislauffähigkeit der Produkte sollen unter anderen folgende Ziele erreicht werden:

- Optimierung der werkstofflichen Verwertbarkeit der Kunststoffe durch Verwendung thermoplastischer Kunststoffe,

- Verringerung der Einzelteile und der Stoffvielfalt,

- Weitestgehender Einsatz von Recyclat für Gehäuse,

- Werkstoffliche Verwertbarkeit,

- Leichte Demontierbarkeit.

Darüber hinaus soll eine Reduktion des Energieverbrauchs in der Gebrauchsphase erzielt werden durch

- Auto-Standby,

- verringerte Verluste im Tonverstärker,

- erhöhten Netzteilwirkungsgrad.

Für den Aufbau elektronischer Geräte aus Metall verfolgt Loewe Opta vor allem folgende Ziele:

- Ressourcenschonung und Schadstoffentfrachtung durch Verwendung von Metallen;

- Gute Verwertbarkeit der Hauptkomponente in der Stahlschrottverwertung bzw. Kupferverwertung.

Philips entwickelt Konzepte, die eine vollständige Wiederverwertung von Bildschirmgläsern erlauben soll. In der ersten Phase werden im industriellen Maßstab die Auswirkungen des Recyclings von Schirm- und Konenglas vom Markt untersucht. Hierbei werden bis zu 30 Prozent Recyclat eingesetzt. In der zweiten Stufe soll eine Standardrezeptur für Schirmglas entwickelt werden, mit dem Ziel, Schirmglas in Schirmglas zu recyceln.

Nokia arbeitet an der Lautsprecheroptimierung durch Anwendung von Finite Elemente Methoden (FEM). Dabei sollen unter anderm für die Magnetsysteme eine 0ptimierung des Wirkungsgrades erzielt werden und neue Magnetmaterialien sowie Magnetgeometrien sollen Ressourcenschonung bringen. 


\section{Ökologische und ökonomische Bewertung}

Die dargestellten Entwicklungsarbeiten der Verbundpartner werden vom Öko-Institut e.V. ökologisch und ökonomisch begleitet. So werden die Neuentwicklungen jeweils im Vergleich zur bestehenden Referenztechnologie auf die erreichbaren Umweltentlastungen hin analysiert und bewertet sowie auf ihre ökonomische Tragfähigkeit hin überprüft. Die ökologische Bewertung folgt dabei im Grundsatz der Methode produktbezogener Ökobilanzen, wobei als wesentliche Ergänzung ein branchenspezifisches Umweltzielsystem erarbeitet wird. Dieses Zielsystem stellt nicht nur den Bewertungsrahmen der ökobilanziellen Untersuchungen dar; es bietet darüber hinaus eine ganzheitliche und langfristige Perspektive, die sich an Umweltpro- blemfeldern und Stoffströmen orientiert und das Leitbild einer nachhaltig zukunftsverträglichen Entwicklung problem- und praxisorientiert konkretisiert. Ein weiterer integraler Bestandteil des Vorhabens ist die Kommunikation der Arbeiten. Das Ziel besteht darin, daß die hier entwickelten Vorstellungen und Technologien durch den akteursübergreifenden Dialog mit Zulieferern, Handel, Entsorgern, Verbrauchern über den engen Teilnehmerkreis hinaus akzeptiert und eingesetzt werden.

\section{Der Autor}

Dr. Volker Strubel ist Dipl. Biologe und Koordinator des Chemiebereichs des Öko-Instituts e.V. Kontakt: Öko-Institut, Postfach 6226, 79038 Freiburg i.Br., Email: strubel@freiburg.oeko.de

\section{Innovationshemmnisse und Fördermöglichkeiten}

\section{Infegierte Umweltfechnik verspricht ökonomische und ökologische Effizienzstei- gerungen. In dem Projekt „Umwelttechnik und wirtschaftliche Entwicklung” des Büros für Technikfolgen-Abschätzung beim Deutschen Bundestag (TAB) ist unter- sucht worden, unter welchen umweltpolitischen Rahmenbedingungen und mit welchen Handlungsansätzen - auch förderpolitischer Natur - diese Chancen genułzł werden könnten.}

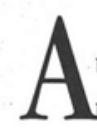
Von Rolf Meyer usgangspunkt der Studie „Umwelttechnik und wirtschaftliche Entwicklung“ des Büros für Technikfolgen-Abschätzung beim Deutschen Bundestag (TAB) ist ein Vergleich additiver und integrierter Umwelttechnik. Dieser Ansatz liefert wertvolle Informationen über deren Wirkungspotentiale, bisher vorherrschende umweltpolitische und unternehmensinterne Rahmenbedingungen und potentielle Handlungsansätze für eine Förderung integrierter Umwelttechnik.

\section{Additive Umwelttechnik}

Dazu wurde additive Umwelttechnik folgendermaßen definiert: Additive Umwelttechnik ist durch einen technologischen Ansatz gekennzeichnet. Mit Hilfe von speziellen Anlagen und
Aggregaten, die bei bestehenden Produktionsprozessen oder Produkten „,eingebaut“ werden, soll dabei die Abgabe von Produktions- oder Konsumtionsrückständen in die Umwelt verhindert oder reduziert werden beziehungsweise Rückstände in eine weniger umweltgefährdende Form überführt werden.

Es erweist sich als Nachteil additiver Umwelttechnik, daß zusätzliche Anlagen zusätzliche Kosten verursachen und damit die Produktivität verringern. Durch den zusätzlichen Einsatz von Energie und anderen materiellen Ressourcen wird zudem zum Umweltverbrauch beigetragen tragen. Und additive Techniken führen oft nur zu Problemverlagerungen in andere Umweltmedien. Integrierte Umwelttechnik hat dagegen das Potential, diese Nachteile zu überwinden bzw. zu verringern.

\section{- Integrierte Umwelttechnik}

Allerdings ist integrierte Umwelttechnik wesentlich schwerer zu definieren. Den vielen vorliegenden Definitionen lassen sich aber Eigenschaften oder Kriterien entnehmen, mit deren Hilfe integrierte Umwelttechnik charakterisiert werden kann:

- Sparsamerer Umgang mit beziehungsweise verringerter Einsatz von Energie und stofflichen Ressourcen in Produktionsprozessen;

- Sparsamerer Umgang mit Energie durch Abwärmenutzung; Produktionsprozeßinternes Recycling oder Kreislauffuihrung (primäres Recycling);

- Verringerung des unvermeidlichen Reststoffanfalls;

- Substitution umweltschädlicher Einsatzstoffe;

- Gänzliche Substitution von Produkten und Produktionsprozessen durch weniger umweltschädliche;

- Weitgehender Verzicht auf End-of-pipe- bzw. additive Technologien;

- Berücksichtigung von Vor- und Folgestufen eines Produktionsprozesses;

- Umweltverträglichere Eigenschaften von Produkten, z.B. Langlebigkeit, Reparaturfreundlichkeit, geringerer Energieverbrauch bei der Nutzung und umweltverträgliche Entsorgung von Produkten

- und Recyclingfähigkeit bzw. umweltverträglichere Entsorgung unvermeidbarer Reststoffe. Der Vergleich der Anwendungsbereiche von integrierter und additiver Umwelttechnik zeigt, daß diese nicht immer als Alternativen, sondern oft auch als sich ergänzende Instrumente zu betrachten sind. Gesamtökologische Optimierungen erfordern in vielen Fällen den gleichzeitigen Einsatz von integrierter und additiver Umwelttechnik.

\section{Innovationshemmnisse für integrierte Umwelttechnik}

Integrierter Umwelttechnik hat sich in den industriellen Investitions- und Innovationsprozessen bisher nur in einem begrenzten Umfang durchsetzen können. Ursachen hierfür sind sowohl in der umweltpolitischen Regulierung (unternehmensexterne Hemmnisse) als auch in den Unternehmen selbst (unternehmensinterne Hemmnisse) zu suchen.

Bei den unternehmensinternen Innovationshemmnissen ist an erster Stelle hervorzuheben, daß der Umweltschutz heute in den Unternehmen noch weitgehend als weniger bedeutend 
(c) 20I0 Authors; licensee IÖW and oekom verlag. This is an article distributed under the terms of the Creative Commons Attribution Non-Commercial No Derivates License (http://creativecommons.org/licenses/by-nc-nd/3.o/), which permits unrestricted use, distribution, and reproduction in any medium, provided the original work is properly cited. 